This item was submitted to Loughborough's Research Repository by the author.

Items in Figshare are protected by copyright, with all rights reserved, unless otherwise indicated.

\title{
Tailoring structure of inclusion with strain-induced closure to reduce Poisson's ratio of composite materials
}

PLEASE CITE THE PUBLISHED VERSION

http://dx.doi.org/10.1063/1.4882855

PUBLISHER

(c) AIP

VERSION

AM (Accepted Manuscript)

\section{PUBLISHER STATEMENT}

This work is made available according to the conditions of the Creative Commons Attribution-NonCommercialNoDerivatives 4.0 International (CC BY-NC-ND 4.0) licence. Full details of this licence are available at: https://creativecommons.org/licenses/by-nc-nd/4.0/

\section{LICENCE}

CC BY-NC-ND 4.0

\section{REPOSITORY RECORD}

Hou, Xiaonan, Hong Hu, and Vadim V. Silberschmidt. 2019. "Tailoring Structure of Inclusion with Straininduced Closure to Reduce Poisson's Ratio of Composite Materials". figshare. https://hdl.handle.net/2134/25631. 


\title{
Tailoring structure of inclusion with strain-induced closure to reduce Poisson's ratio of composite materials
}

\author{
Xiaonan Hou, ${ }^{1} \mathrm{Hong} \mathrm{Hu},{ }^{1, a)}$ and Vadim Silberschmidt ${ }^{2}$ \\ 1 \\ Institute of Textiles and Clothing, The Hong Kong Polytechnic University, Hung Hom, Hong Kong \\ Wolfson School of Mechanical and Manufacturing Engineering, Loughborough University, \\ Loughborough LE1 1 3TU, United Kingdom
}

\begin{abstract}
A novel 3D continuum shell structure is introduced as inclusion for composite materials with special mechanical properties in this paper. Its geometry is based on a hollow re-entrant tetrahedron. In a composite, such an inclusion can demonstrate a closure effect induced by external compression. Its specific deformation mechanism results in a special character of deformation and affects effective (global) mechanical properties of the composite. A finite-element method is used to explore quantitatively and qualitatively the deformation mechanism of the suggested inclusion and its effect on the overall mechanical performance of the composite. In this study, geometrical features of the inclusion are used as parameters. The obtained results demonstrate that this kind of inclusion could reduce the composite's Poisson's ratio; moreover, its magnitude is adjustable by changing geometrical parameters of the inclusion. Besides, an overall hardening effect is achieved for the composite, with the magnitude of global stiffness also significantly affected by geometrical features of the inclusion. Thus, the developed inclusion actually provides a potential to develop new composites with a tunable Poisson's ratio and enhanced mechanical properti@.
\end{abstract}

\section{INTRODUCTION}

With development of modern engineering, requirements to structural materials are becoming more specific and stringent. For centuries, many efforts have been made to improve mechanical properties of engineering materials. There are two major factors defining mechanical properties of composites: properties of constituents and microstructure. Recently, the concept of mechanical metamaterials has been introduced, ${ }^{1}$ which places a stronger emphasis on the effects of the special microstructure of the material on its overall properties and performance. The recent developments illustrate that engineering materials with novel, tailored mechanical properties could be developed from a design concept by manipulating the constituents and microstructures. As a consequence, there are many studies on development of new structures with unusual behaviours and material properties. One research focus is to develop structures with a shrinking effect and structural instability. ${ }^{2-6}$ In engineering, these concepts are widely used for drug delivery, ${ }^{7}$ optical devices, ${ }^{8}$ and sensors. ${ }^{9}$ Especially, when a structure with a volumeshrinking effect is used as an inclusion in a composite structure, a series of special material properties could be achieved. ${ }^{10,11}$

One of the seminal studies is the Lakes's theoretical analysis of a conceptual composite with extreme damping and a negative Poisson's ratio (NPR). ${ }^{10}$ In his study, a concept of a composite system with perfect inclusions ${ }^{10}$ is

\footnotetext{
a) Author to whom correspondence should be addressed. Electronic mail: tchuhong@polyu.edu.hk.
}

proposed. The analysis demonstrates that the Poisson's ratio of a composite structure could be reduced from positive to negative, when the volume of its inclusions reduces as it closes. Besides, according to the classic theory of solid mechanics, this sort of materials potentially exhibits other specific mechanical properties ${ }^{12-16}$ induced by the NPR, such as enhanced levels of indentation resistance, ${ }^{17}$ shear stiffness, ${ }^{17-19}$ fracture toughness, ${ }^{20,21}$ and energy-absorption ability. ${ }^{20,22,23}$ However, the research stopped at the theoretical stage because the inclusion with closure effect is hard to implement in real structural materials.

At present, only a few studies have successfully introduced the volume-closure effect into a 3D structure. One of such structures is a $3 \mathrm{D}$ graded structure developed as a vehicle blast-protector. ${ }^{20}$ Its basic unit is a frame structure based on a pyramid polyhedron with one re-entrant face. When the structure is exposed to external loading, it contracts (densifies), and the internal void space is closed. The last development is a 3D shell structure, known as Buckliball. ${ }^{3,24}$ It is a continuous shell structure, which could exhibit an encapsulation effect induced by buckling under pressure loading. The deformation mechanism seems to meet the requirements, as expected by Lakes. ${ }^{10}$ But there are still some limitations: the deformation mechanism for a single Buckliball is defined by the closure of holes in its surface that ideally requires hydrostatic loading conditions. As a result, the area of surface of the deformed Buckliball decreases during the deformation. When it is embedded in a matrix of a composite, the surface holes of the Buckliball cannot close because of the constraints imposed by the matrix material. Therefore, the Buckliball can hardly be 
used as an inclusion in a composite or transfer its specific properties to it.

In this paper, a concept of a $3 \mathrm{D}$ re-entrant hollow polyhedral structure with a strain-induced closure effect is introduced as inclusion for composites. The study starts from an analysis of different deformation mechanisms of inclusions linked to their frame and shell. Based on the obtained results, an inclusion derived from the tetrahedron is suggested. It could close its internal void even with constraints from matrix material and external loading, affecting the overall mechanical properties of the composite. To evaluate the effects of this inclusion on the composite, a representative element of the composite is developed at this stage of the study, exhibiting a tunable Poisson's ratio and enhanced stiffness. The element consists of only one inclusion and the matrix. The inclusion is supposed to be fabricated using a stiffer material to act as a reinforcing element of the composite. Thanks to its geometric features, it undergoes a straininduced structural encapsulation under compressive loading, exhibiting the volume-closure effect. The specific deformation mechanism of the inclusion leads to a reduction of the global Poisson's ratio and enhanced stiffness of the composite. The finite-element method was employed to analyse compression behaviour of this composite to validate its conceptual design. In addition, the effects of geometrical features of the inclusion on the mechanical performance of the overall composite structure are also evaluated.

\section{INCLUSION}

The proposed composite comprises two components: a matrix and an inclusion. The matrix is the basic material of the composite with an internal polyhedral void. The inclusion is embedded in the void in the matrix, inducing closure of the void, and mainly determines the deformation mechanism of the composite. In this study, a re-entrant tetrahedron (Fig. 1(b)) is chosen as the shape of both the void in the matrix and the inclusion of the composite, since the tetrahedron is the simplest polyhedron (Fig. 1(a)) and representative. The length of the edges $L$ is 10 , and the faces of the tetrahedron are re-entrant from the central points of the faces towards its internal space. The degree of re-entrant face is controlled by the distance $(H)$ from the vertexes $(e, f, g$, and $h)$ to their corresponding faces (Fig. 1(b)). It is defined as 0.5 at this stage of analysis. To introduce structure instability to the void, all the edges face inward, with a very small degree. There are two types of edges: outline and re-entrant. The outline edge is the original edge of the tetrahedron, such as edge $c-d$. The middle point of edge $c$ - $d$ indents the tetrahedron inwards by a very small value of $L_{i}=0.05$ along the perpendicular in the plane defined by vertexes $c, d$ and $e$. The re-entrant edge is the edge, which defines the re-entrant face of the tetrahedron, such as edge $d$ - $g$. To introduce curvature, the middle point of edge $d$ - $g$ indents the tetrahedron inwards by $L_{i}=0.05$ along the perpendicular in the plane defined by points $a, d$, and $g$.

To develop a composite with a reduced Poisson's ratio and enhanced mechanical properties, the inclusion should exhibit two specific mechanical properties: an effect of volume closure and structural concentration. Inspired from the successful 3D NPR structures, ${ }^{3,20,21}$ two different approaches are adopted for designing the inclusion: a re-entrant frame inclusion and a re-entrant shell inclusion. The former is defined using the edges of the re-entrant tetrahedron (Fig. 1(b)), while the latter using the faces of the re-entrant tetrahedron (Fig. 1(b)). To evaluate deformation mechanisms of different types of inclusions in composites, finite-element models were developed by embedding these two suggested inclusions in the same matrix; the assembly scheme is shown in Fig. 2. To eliminate the effects of boundary conditions of the composite on the deformation of the embedded inclusion, a relatively large cubic matrix element with dimensions $30 \times 30 \times 30$ was used (Fig. 2(a)). A void is located in the matrix; its geometric centre coincides with that of the composite element. The shape and dimensions of the void and the inclusion are the same as those of the basic re-entrant tetrahedron (Fig. 1(b)). A composite element is assembled by embedding the inclusion into the matrix (Fig. 2(c)). The boundary conditions used in simulations corresponded to the fixture of a specimen in the real-life test. The bottom of the structure was fixed (using "ENCASTRE" option in ABAQUS with all the degrees of freedom of the surface constrained: $\mathrm{U} 1=\mathrm{U} 2=\mathrm{U} 3=\mathrm{UR} 1=\mathrm{UR} 2=\mathrm{UR} 3=0$ ). A uniform displacement in the negative $y$ (U2) direction was applied on the top surface of the structure to simulate the static

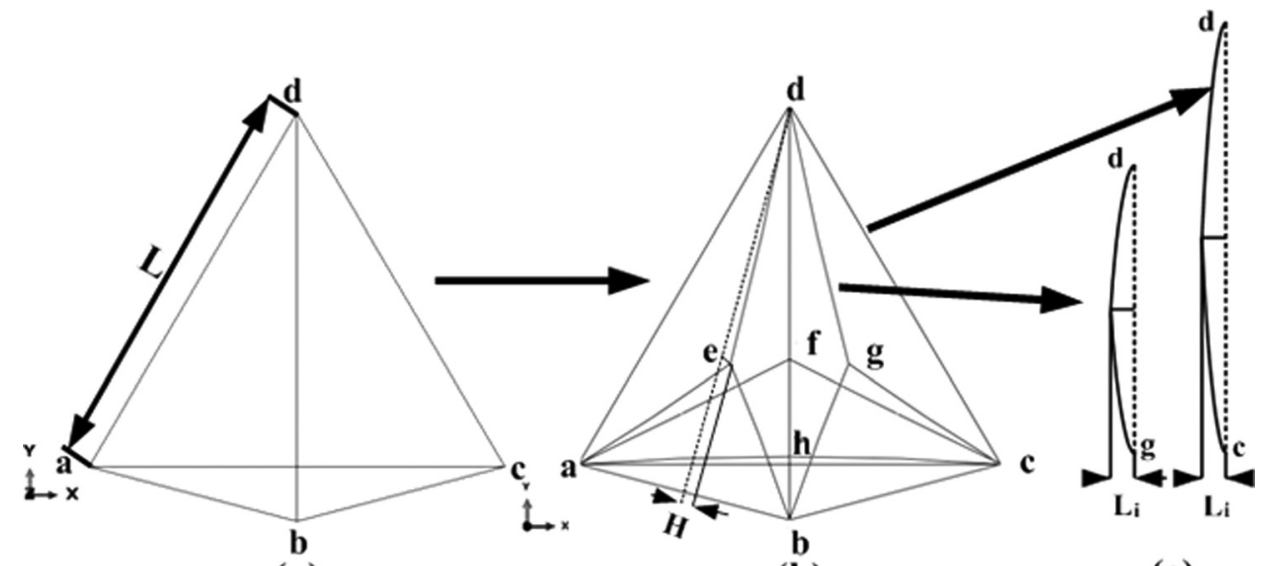

(a) (b)
FIG. 1. Geometry model for void: (a) basic tetrahedron; (b) re-entrant tetrahedron; (c) details of re-entrant edges. 


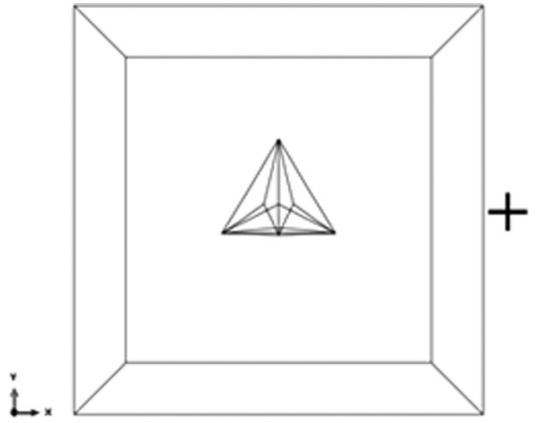

(a)

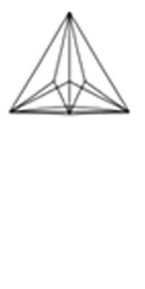

(b)

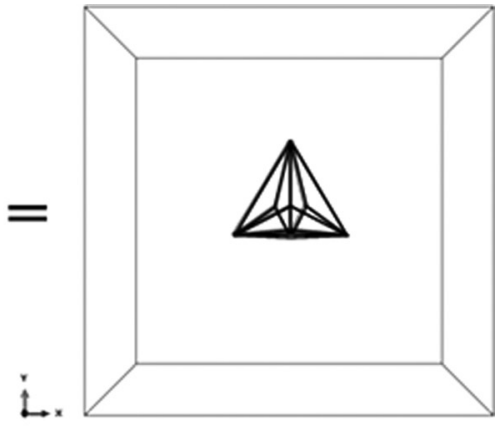

(c)
FIG. 2. Scheme of assembly of composite element: (a) matrix with void; (b) inclusion; (c) composite structure. compressive deformation. Contacts between the internal surfaces of the inclusion were allowed in simulations.

For the model with the frame inclusion, the cross section of the frame is circular, with diameter of 0.4 . The elastic modulus of the matrix was defined by a given value $A$, and its Poisson's ratio was 0.3 . The elastic modulus for the frame inclusion was defined as $100 \mathrm{~A}$, and the Poisson's ratio was also 0.3. Elements B31 (ABAQUS) were used to mesh the frame and element C3D10 was used for the matrix. For the model with the shell inclusion, the matrix was the same as in the previous model; thickness of the shell was defined using a parameter $T$, which was 0.2 at the initial stage of the study. It is offset from the bottom of the faces of the void. The elastic modulus 100 A was used for the shell, and element S4R was used to mesh the inclusion. The Poisson's ratio of the shell was 0.3 .

Figure 3 demonstrates deformation patterns obtained in simulations for the models with the frame and shell inclusions, respectively. It is apparent that two types of inclusions induce different deformation mechanisms. For the composite element with the frame inclusion (Fig. 3(a)), the void flattened when a compressive load was applied and the frame of the inclusion collapsed. Even though the void is compressed thanks to the re-entrant geometry of both inclusions, the deformation process does not result in lateral contraction and strain-induced concentration, which would be exhibited by a re-entrant frame without any constraints by the matrix.

However, the composite structure with the shell inclusion (Fig. 3(b)) demonstrated a different deformation pattern. All its four faces shrank towards the centre of the void with the increasing overall strain (Fig. 4), exhibiting both volume closure and structural concentration effects. Based on this deformation mechanism, the matrix material is dragged by the re-entrant inclusion into the void, closing it. As shown in Figure 5, the volume of the void decreases with the increasing external compressive strain. The initial void volume of the composite element before the start of compression was 81.62. It decreased to 75.17 , when the overall compressive strain of $5 \%$, i.e., $7.9 \%$ of it was occupied by the compressed matrix material. When overall strain reached $29.6 \%, 65.16 \%$ of void is compressed due to the shrink of all four re-entrant faces of the inclusion. Comparing this development with the deformation pattern of the frame inclusion, the shell inclusion exhibited the apparent volume-closure and structural concentration effects rather than the flattening effect of the latter inclusion. Therefore, the re-entrant-shell inclusion was
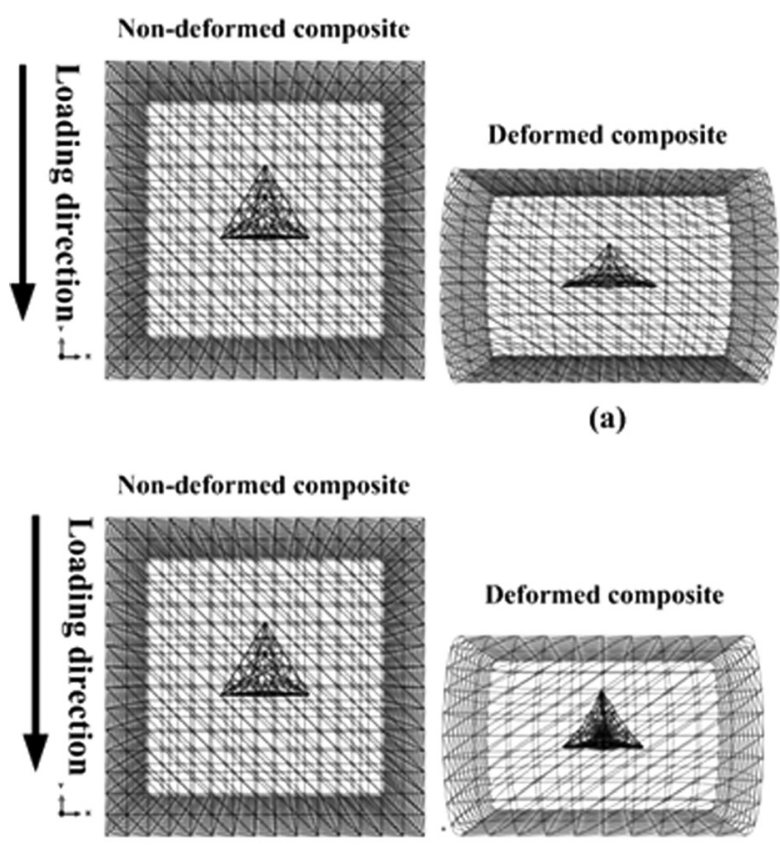

(a)

Deformed frame inclusion

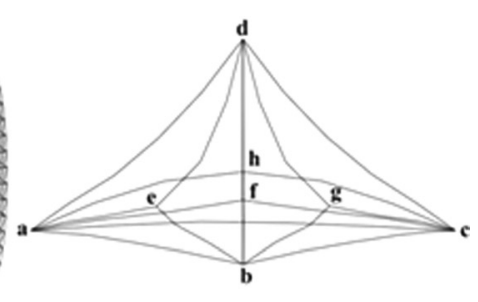

Deformed shell inclusion
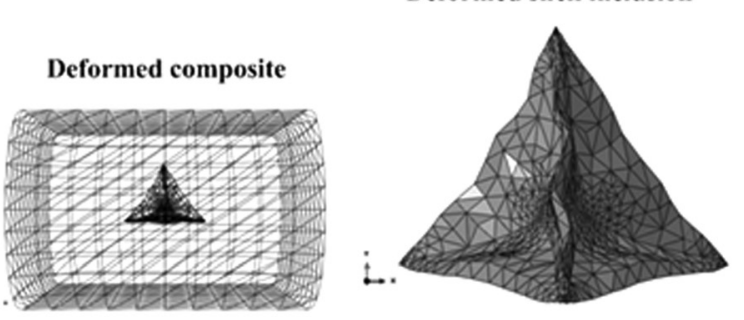

FIG. 3. Deformation of composite elements with frame (a) and shell (b) inclusion.

(b) 


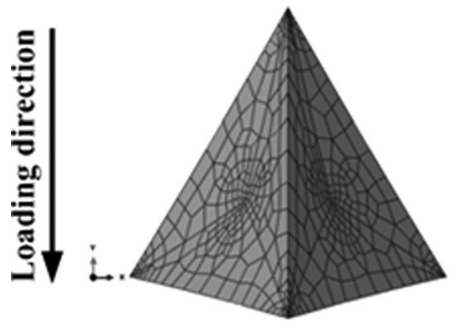

(a) Front view of inclusion (strain: 0\%)

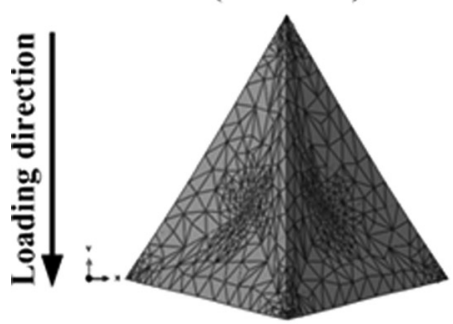

(b) Front view of inclusion (strain: 10\%)

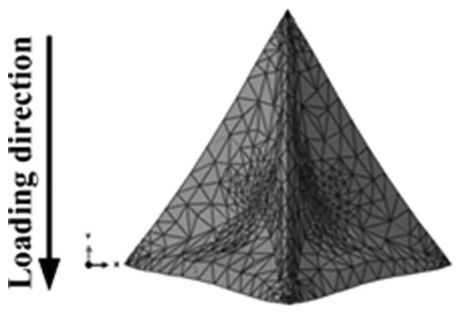

(c) Front view of inclusion (strain: $20 \%$ )

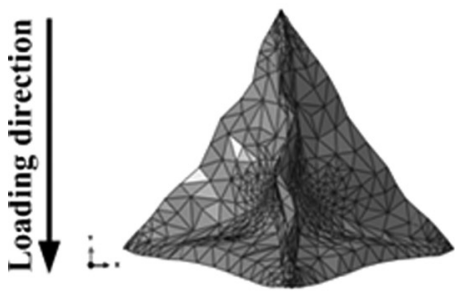

(d) Front view of inclusion (strain: $\mathbf{2 9 . 6 \% )}$

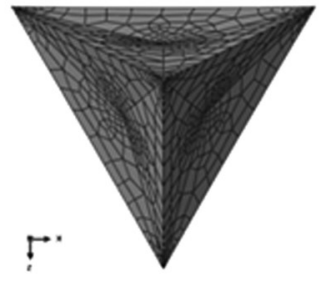

Top view of inclusion (strain: $0 \%$ )

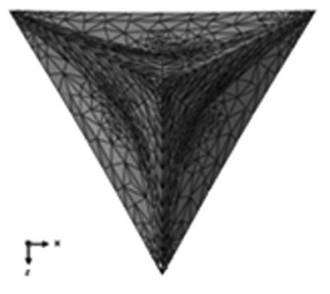

Top view of inclusion (strain: $10 \%$ )

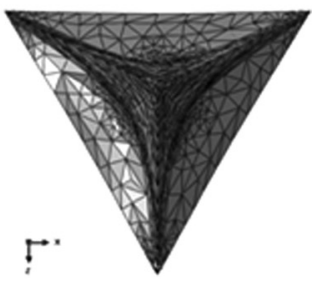

Top view of inclusion (strain: $\mathbf{2 0} \%$ )

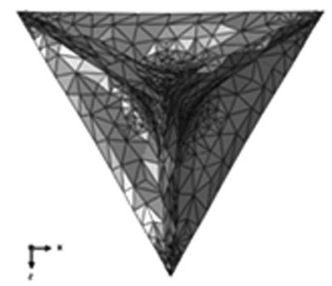

Top view of inclusion (strain: $\mathbf{2 9 . 6 \%}$ )

FIG. 4. Deformation mechanism of shell inclusion at various levels of external compressive strain: (a) $0 \%$; (b) $10 \%$; (c) $20 \%$; (d) $29.6 \%$.

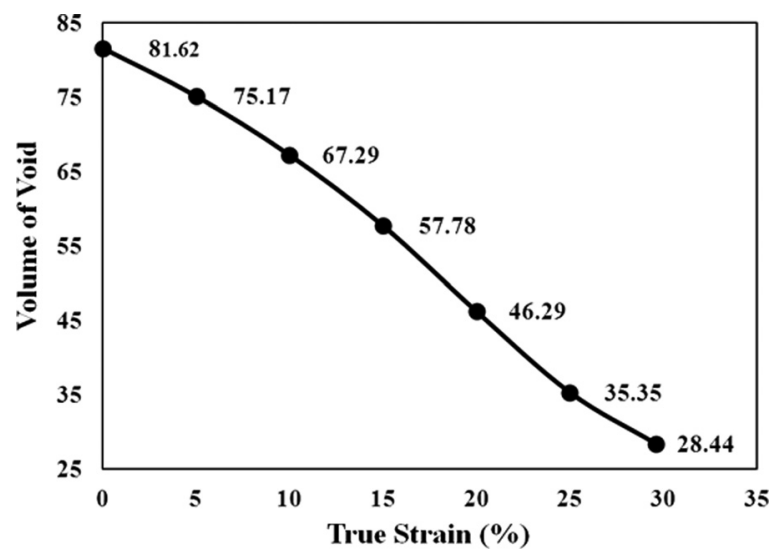

FIG. 5. Void volume reduction under different compressive strain levels.

determined as the inclusion for the further development of the composite with a special type of mechanical behaviour.

\section{COMPOSITE ELEMENT WITH INCLUSION}

Based on the above analysis, the hollow re-entrant shell inclusion was selected to develop a representative composite element. In order to investigate the effect of this inclusion on mechanical performance of the composite, a cylinder-shaped matrix with relatively small dimensions was chosen for the composite element (Fig. 6). The circular cross section of the element reduces the effect of the tetrahedral shape of the inclusion, and the element's relatively small dimensions allow evaluation of the effects of the inclusion on the overall mechanical properties. The initial diameter of the cross section $\mathrm{D}_{0}$ was 12 , its height 16 . The inclusion was placed in the void of the cylinder; the assembly scheme assured that the inclusion was located in its centre (Fig. 6(c)). The effects of two geometrical parameters - a degree of re-entrant faces and thickness of the shell of inclusion-on the composite's behaviour were analysed.

To analyse the effect of the degree of re-entrant faces, three finite element models were developed: model-05-02, model-04-02, and model-03-02. The codes of the models reflect different levels of the inclusion's geometrical parameters; the notation is "model-H-T," where $H$ is the degree of re-entrant faces of inclusion and $T$ is the thickness of the shell of inclusion. In this study, thickness of the shell was constant $(T=0.2)$, while three values were assigned to the degree of re-entrant faces $H: 0.5,0.4$, and 0.3 . The volume fractions of the components for the resulting composite elements are shown in Table I. The mesh, material definitions, and boundary conditions of the models are in accord to the FE (Finite Element) model presented in Sec. II.

Typical compression behaviour of this kind of composite element is shown in Fig. 7. When a compressive loading was applied (Fig. 7(b)), its lateral surface contracted along the transversal direction. Apparently, it demonstrated a different deformation mechanism from that for conventional materials that usually extend laterally under compression. The phenomenon is due to the closure effect of the embedded inclusion (Fig. 7(c)), with the matrix material being dragged inwards the void space.

However, for different degrees of the re-entrant faces, deformations of composite elements are quantitatively different. The change in the Poisson's ratio of the studied composite structures is presented in Fig. 8. The results were calculated using the initial diameter of the cylinder $D_{0}$ and the diameter of the middle cross sections $\left(D_{1}\right)$ of the deformed element (Fig. 7(b)). Obviously, the Poisson's ratios of the composite elements are much lower than the Poisson's ratio of their constituent materials, which was 0.3 . For strains below $18 \%$, nearly constant levels were obtained. This can be explained by a continuous void closure in the composite elements (Fig. 9(a)) with the increasing compressive strain. Similar deformation patterns were demonstrated in all of three composite elements. However, the elements with lower re-entrant degrees $H$ demonstrate higher Poisson's ratios at overall strain below 18\%: e.g., the average Poisson's ratio of 


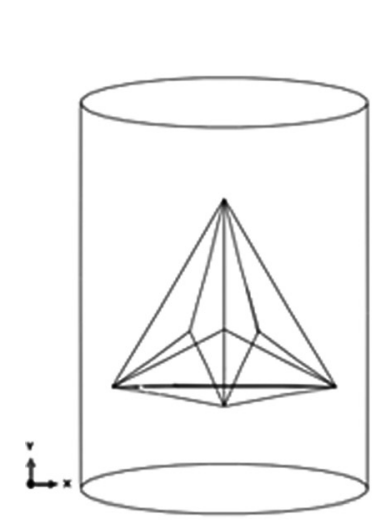

(a)

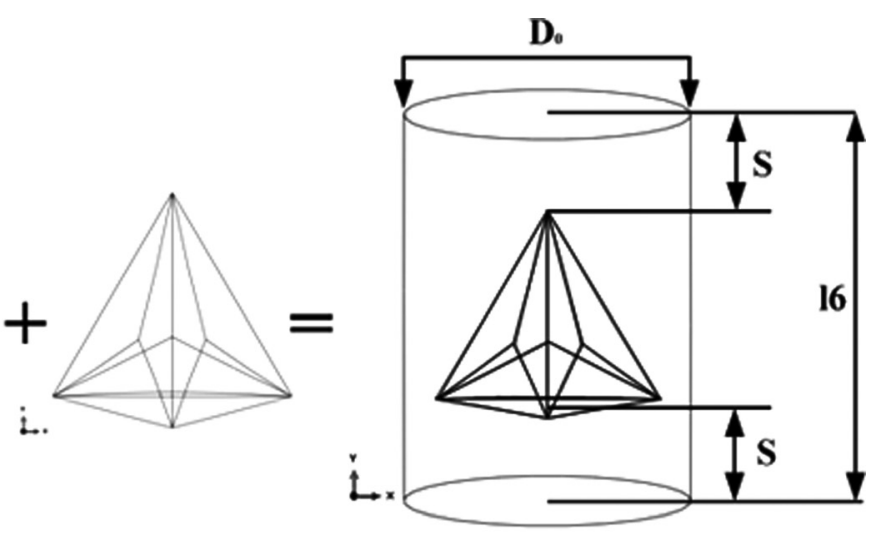

(c)
FIG. 6. Scheme of assembly of cylindrical composite element: (a) matrix with void; (b) inclusion; (c) composite structure.

TABLE I. Volume fractions of components of composite elements.

\begin{tabular}{lrrrrr}
\hline \hline Model code & $0.5-0.2$ & $0.4-0.2$ & $0.3-0.2$ & $0.3-0.3$ & $0.3-0.4$ \\
\hline Volume fraction of void $\left(\mathrm{R}_{\mathrm{v}}\right) \%$ & 4.51 & 4.82 & 5.14 & 5.14 \\
Volume fraction of shell of inclusion $\left(\mathrm{R}_{\mathrm{s}}\right) \%$ & 1.91 & 1.90 & 5.14 & 5.14 \\
Volume fraction of matrix $\left(\mathrm{R}_{\mathrm{m}}\right) \%$ & 93.58 & 93.28 & 92.97 & 9.74 & 92.02 \\
\hline \hline
\end{tabular}

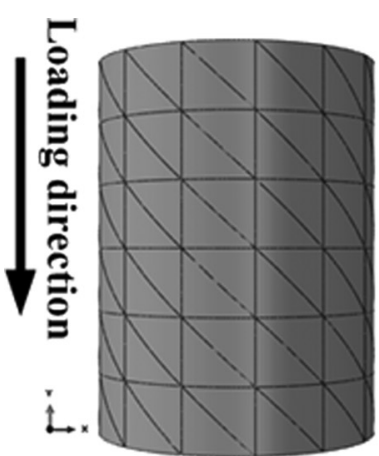

(a)

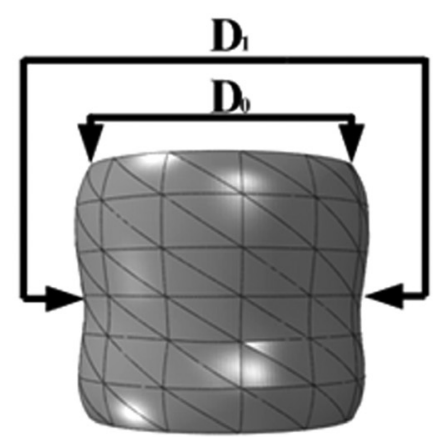

(b)

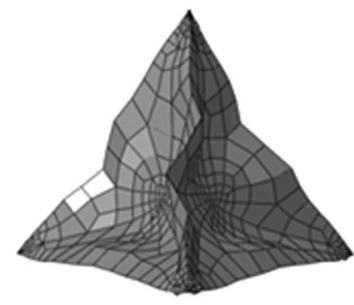

(c)
FIG. 7. Typical deformation pattern of cylinder composite element (model05-02) with shell inclusion: (a) nondeformed composite; (b) deformed composite element; (c) deformed shell inclusion.

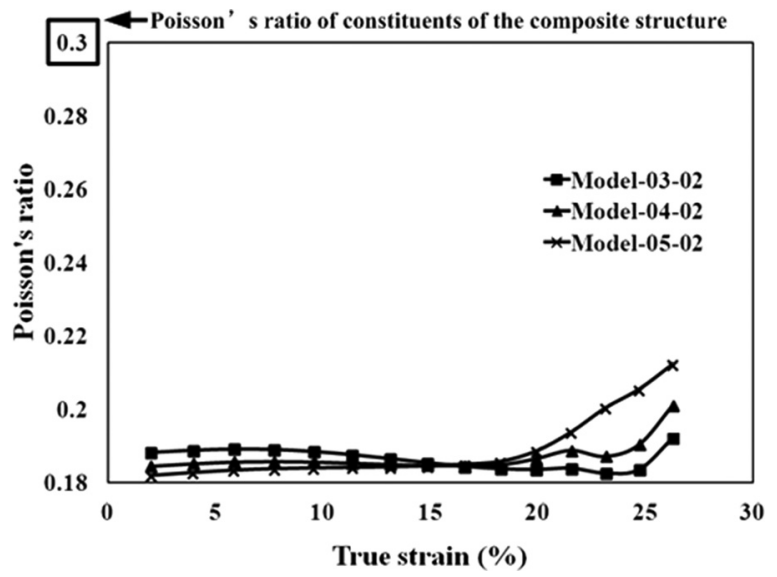

FIG. 8. Evolution of Poisson's ratio with strain for composite elements with different degrees of re-entrant faces. the composite element with the smallest $H$ (model-03-02) is $1.79 \%$ higher than the one for the composite with the highest $H$ (model-05-02). Further compression changed the trends. For instance, the value of the Poisson's ratio obtained for model-05-02 at strain of $26.2 \%$ was $14.3 \%$ higher than that strain of $20 \%$. For the composite elements with lower $H$ model-04-02 and model-03-02-the respective increases were $8.7 \%$ and $4.6 \%$. These results demonstrate that composite elements with smaller values of $H$ have more stable values of the Poisson's ratio. According to Table I, such elements have a higher volume of void. It means that void closure continues even at higher strain levels as seen in Fig. 9(a).

However, the decline of void volume with strain is nonlinear due to the geometry of inclusions (Fig. 9(a)). To link the change in void volume with deformation of the composite element, a ratio $\mathrm{R}$ is introduced to define the speed of these processes

$$
\mathrm{R}=-\Delta \mathrm{R}_{\mathrm{v}} / \Delta \varepsilon
$$




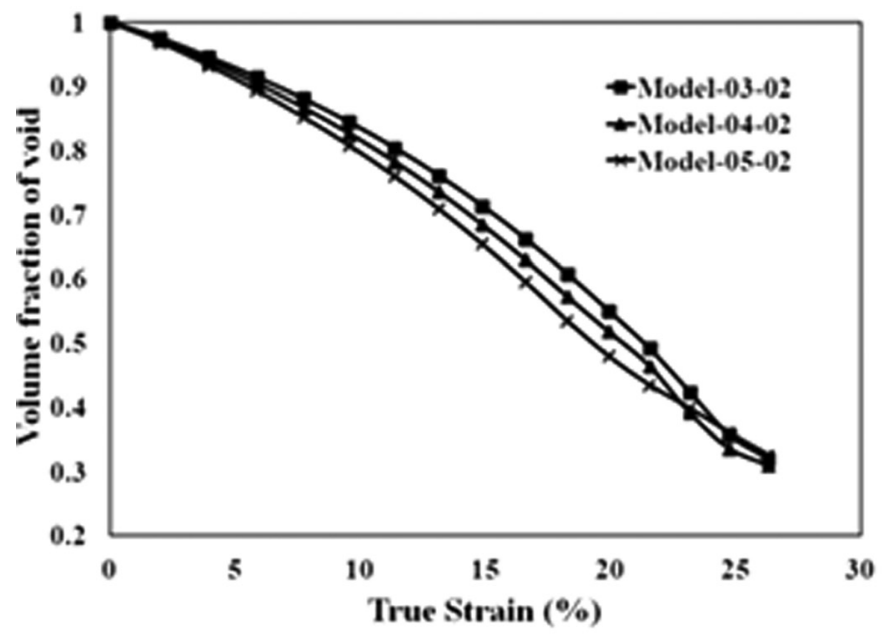

(a)

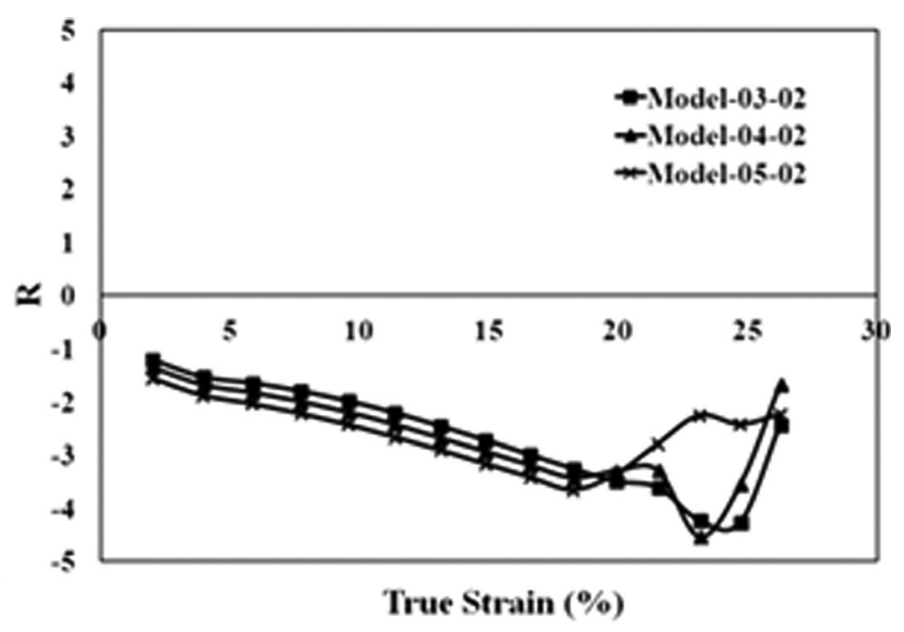

(b)

FIG. 9. Evaluations of volume fraction of void (a) and R (b) with strain for composite elements with different degrees of re-entrant faces.

where $\Delta \mathrm{R}_{\mathrm{v}}$ is the change in volume fraction of void and $\Delta \varepsilon$ is the respective change in overall true strain of the composite element.

The evolution of this ratio with compressive strain is presented in Fig. 9(b), which provides a better understanding of the results shown in Fig. 8. When the overall strain level is below $18 \%$, a nearly linear decrease is obtained for all three composite elements, reflected in nearly stable Poisson's ratios (Fig. 8). Apparently, the composite with higher $H$ has a higher absolute value of $R$, which explains that lower values of its Poisson's ratio. At overall strains exceeding $18 \%$, the ratio $R$ demonstrated irregular changes with increasing strain, resulting in fluctuations of the Poisson's ratios at a relatively large scale. It is caused by a very complicated closure procedure of the inclusions at such high strain levels. Their internal surfaces start to contact each other, and local buckling occurs in certain parts (Fig. 7(c)).

Another important geometrical parameter of the studied inclusion is thickness of the shell, which may affect mechanical properties of the overall composite. Thus, four different FE models were developed to simulate the composite elements with different inclusions, which have the same degree of reentrant face $(0.3)$ and different thicknesses $(0.2,0.3,0.4$, and $0.5)$. The volume fractions of three components of the composite elements are shown in Table I. The volume fraction of void was kept constant in all three composites, since a constant value $(5.14 \%)$ was chosen for the degree of re-entrant face.

The calculated stress-strain relationships of the composite elements are presented in Fig. 10(a). Although the defined material properties of the matrix and shells of the composite elements are linear elastic, the composite elements show nonlinear overall mechanical responses. Obviously, the composite with the inclusion with a thicker shell is stiffer: at strain of 25\%, the level of stress obtained for model-03-05 (highest $T$ ) was $2.8 \%$ higher than that model-03-02 (lowest $T$ ).

To evaluate the nonlinear mechanical performance of the composite elements, their instantaneous moduli were defined. Figure 10(b) shows the evolution of the composites' moduli with strain, demonstrating significant stiffening with increase in compression strain.

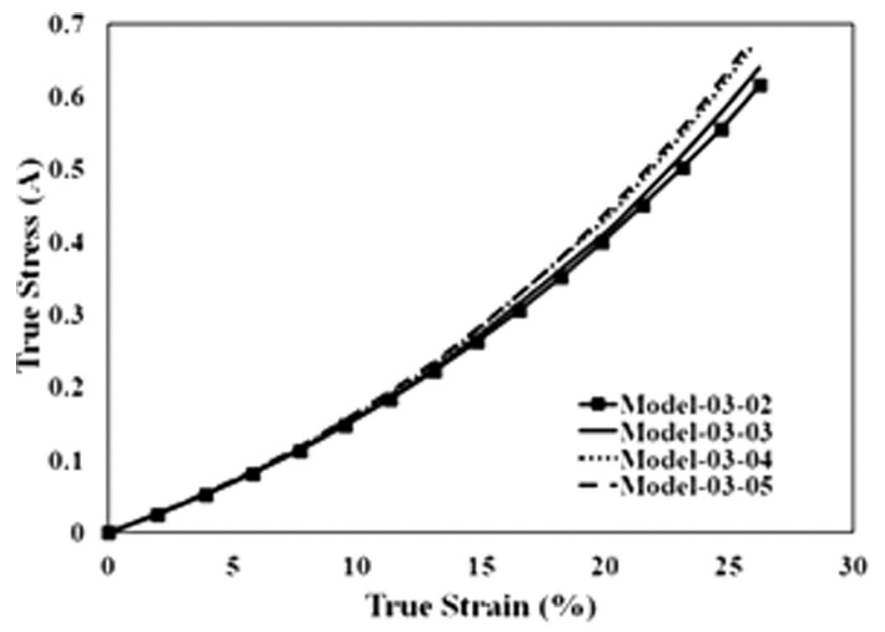

(a)

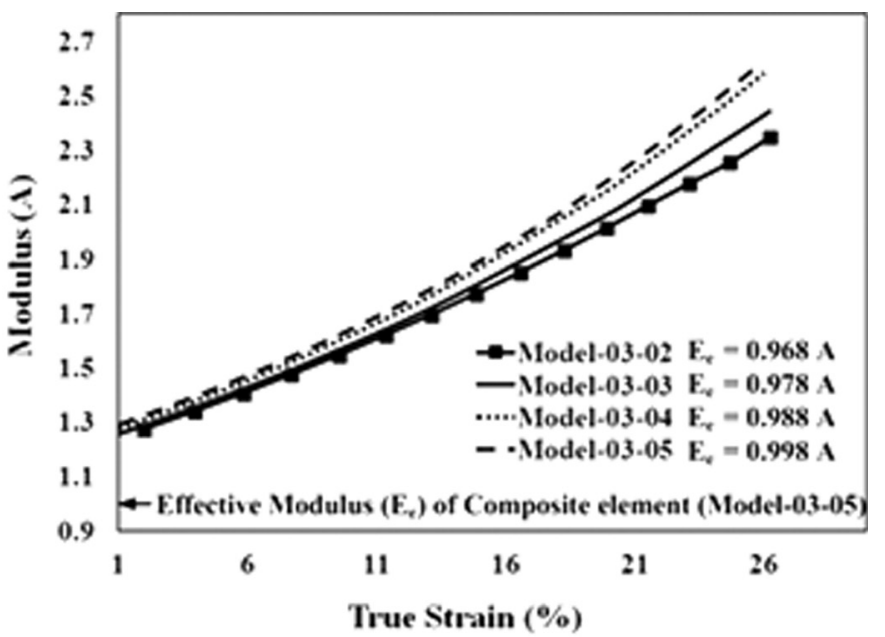

(b)

FIG. 10. Stress-strain relationships (a) and overall moduli (b) with strain for composites with different thickness of shells. 
To obtain a better understanding of the hardening effect, an effective modulus is introduced to define the global stiffness of the composite elements, including their voids. In the suggested composite elements, the overall volume consists of volume of shell of inclusions $\left(\mathrm{V}_{\mathrm{s}}\right)$, volume of voids $\left(\mathrm{V}_{\mathrm{v}}\right)$, and volume of matrix $\left(\mathrm{V}_{\mathrm{m}}\right)$. So, the effective modulus was determined based on the theory of the rule of mixtures by considering the effect of voids.

First, it was assumed that there is a continuous composite material with only two constituents: materials of shell of inclusion and matrix. It has the same volume $\mathrm{V}^{\prime}$ as the developed composite element, which is given by

$$
\mathrm{V}^{\prime}=\mathrm{V}_{\mathrm{s}}+\mathrm{V}_{\mathrm{m}}
$$

For this two-phase composite, the low-bound modulus of this composite $\mathrm{E}^{\prime}$ could be easily calculated with the rule of mixtures

$$
\mathrm{E}^{\prime}=\mathrm{E}_{\mathrm{s}} \mathrm{E}_{\mathrm{m}} /\left(\mathrm{E}_{\mathrm{s}} \mathrm{V}_{\mathrm{m}} / \mathrm{V}^{\prime}+\mathrm{E}_{\mathrm{m}} \mathrm{V}_{\mathrm{s}} / \mathrm{V}^{\prime}\right)
$$

where $E_{\mathrm{s}}$ is the elastic modulus of the material of the shell of inclusions and $\mathrm{E}_{\mathrm{m}}$ is the modulus of the matrix. The account for voids can be introduced in a way, traditional for low-level porosity or damage. According to this, the effective stiffness of such a material is normalised by the parameter $(1-\mathrm{D})=\left(1-\mathrm{V}_{\mathrm{v}} / \mathrm{V}\right)$, where $\mathrm{V}=\mathrm{V}^{\prime}+\mathrm{V}_{\mathrm{v}}$ is the total volume of the material. Hence, the effective Young's modulus of the three-phase composite (matrix, inclusions and voids) obtained with the two-step procedure has the following form:

$$
\mathrm{E}_{\mathrm{e}}=\mathrm{E}^{\prime} \mathrm{V}^{\prime} /\left(\mathrm{V}^{\prime}+\mathrm{V}_{\mathrm{v}}\right)
$$

Fig. 10(b) shows the effective modulus $\mathrm{E}_{\mathrm{e}}$ of the composite elements calculated with Eq. (4). Its magnitude for all the composite elements is lower than the modulus (A) of matrix materials caused by the presence of voids that is not even compensated by the reinforcing component (shell of voids) due to its low volume fraction.

However, all the moduli calculated in numerical simulations for the developed composite elements are higher than the effective modulus (Fig. 10(b)), indicating that a proper design of microstructure of a composite could reduce the negative effect of voids on the overall stiffness. When the strains reach $1 \%$, the moduli of all the composite elements are higher than $1.2 \mathrm{~A}$, i.e., already $20.24 \%$ higher than the effective modulus $(0.998 \mathrm{~A})$ of the composite element (model-0.3-0.5), which exhibits the highest effective modulus among all the evaluated cases. For strains exceeding $25 \%$, the moduli increase to the values higher than $2 \mathrm{~A}$, showing a significant hardening effect.

\section{DISCUSSION AND CONCLUSIONS}

A novel 3D inclusion is developed for composites, which could exhibit a tunable Poisson's ratio and enhance their mechanical properties. The study starts from design of the inclusion to achieve two special effects in terms of its deformation mechanism: a void-closure effect and an effect of structural concentration. Then, composite elements are developed based on the suggested inclusion. Using the finiteelement method, the deformation mechanism caused by inclusions with various geometrical parameters and their effects on the overall mechanical performances of respective composite elements are analysed. Based on the obtained results, the following features can be discussed and conclusions drawn:

1. The design of inclusion was inspired by the well-know reentrant NPR structures. However, the current research into NPR structures is focused mainly on frames. Although such structures could exhibit the NPR effect and some specific mechanical performances, they could not be used in composites as reinforcing elements because deformation of frames cannot initiate similar deformation of the matrix material in a composite material. Thus, the inclusion has to be developed on the basis of a continuum shell, encapsulating a void space. Within a composite element, the shell structure contracts inward the void space and exhibits the volume-closure effect. For the inclusion developed in this study, more than $65 \%$ of the original void space would be closed (and the effect could be enhanced by defining its parameters properly), which means that the closure effect is very effective. Since the shell of the inclusion is bonded with the matrix material, the latter was dragged by the shell filling the void space. Consequently, the composite tended to contract; this resulted in specific overall mechanical performances: reduction in the Poisson's ratio and the hardening effect. In this study, the inclusion was designed on the basis of tetrahedron, which was treated as a simple example for validation of the concept of the inclusion closure.

2. The composite element with the developed inclusion exhibited specific mechanical performance. First, the overall Poisson's ratio of the composite was significantly lower than that of its constituents. Some lateral contraction of the composite element can be observed in the deformed FE models, when a compressive load was applied. A relatively stable Poisson's ratio with strain increase was obtained; it was due to a nearly linear change in the ratio $R$ of the inclusion. The composite element with higher $H$ values had a lower magnitude of the Poisson's ratio at this stage of deformation since the void in this composite could be closed quicker. At relatively high overall strains, the ratio $R$ changed irregularly, and the composite's Poisson's ratio increased significantly. It can be explained by a very complex deformation at this stage, characterised by final closure of the void, contacts between its internal surfaces and buckling of the shells. However, the composite element with lower $H$ generally showed a more stable performance, since, thanks to a bigger void space within the inclusion, contact and buckling occurs later than in the composite with higher $H$.

3. Besides the special deformation character, a hardening effect is observed in the composite with the designed inclusion. Although the material properties of the constituents are linear elastic, a nonlinear global mechanical behaviour was obtained, with the modulus increasing with 
the overall strain. It means that the stiffness of composite was enhanced. Moreover, the geometrical parameter $T$ was proved to be an important factor affecting the hardening effect for given material properties of the constituents. A higher magnitude of $T$ resulted in higher overall stiffness of the composite element, in accordance with the classic rule of mixture.

The most important contribution of this study is to provide a possible approach to design composites with a tunable Poisson's ratio and enhanced mechanical properties, for example: light weight composite materials with relative higher impact resistance and shear stiffness. According to the analysis of this study, the developed inclusion exhibits both structural concentration and volume-closure effects. The composite element with this inclusion demonstrated an effective reduction in the Poisson's ratio, tunable by adjusting geometrical parameters of the inclusion. Moreover, the hardening effect of the composite structure was validated that was also affected by the inclusion's geometry features. Although the discussed effects were relatively small in the studied composite elements with one inclusion, it is mostly due to low volume fractions of void in them-some $5 \%$. For the future study, there are two methods, which could enhance these effects. First, inclusions on the basis of polyhedrons with higher orders of faces will be analysed to increase the volume fraction of voids in a composite. Second, the fraction of void could be also increased by increasing the number of inclusions. Actually, a similar concept was used in our previous study. ${ }^{25}$ In it, a $2 \mathrm{D}$ composite structure with a large number of hollow re-entrant inclusions demonstrated specific mechanical performances in terms of both its Poisson's ratio and overall stiffness. These results will be used to extend our previous study from 2D to 3D.
${ }^{1}$ J. Lee, J. P. Singer, and E. L. Thomas, Adv. Mater. 24, 4782 (2012).

${ }^{2}$ B. Li, F. Jia, Y. Cao, X. Feng, and H. Gao, Phys. Rev. Lett. 106, 234301 (2011).

${ }^{3}$ J. Shim, C. Perdigou, E. R. Chen, K. Bertoldi, and P. M. Reis, Proc. Natl. Acad. Sci. U.S.A. 109, 5978-5983 (2012).

${ }^{4}$ F. Caruso, R. A. Caruso, and H. Mohwald, Science 282, 1111 (1998).

${ }^{5}$ W. H. Suh, A. R. Jang, Y. H. Suh, and K. S. Suslick, Adv. Mater. 18, 1832 (2006)

${ }^{6}$ O. A. Goussev and P. Richner, J. Appl. Phys. 88, 4013 (2000).

${ }^{7}$ Y. Zhu, J. Shi, W. Shen, X. Dong, J. Feng, M. Ruan, and Y. Li, Angew. Chem. Int. Ed. 44, 5083 (2005).

${ }^{8}$ E. Hao, S. Li, R. C. Bailey, S. Zou, G. C. Schatz, and J. T. Hupp, J. Phys. Chem. B 108, 1224 (2004).

${ }^{9}$ C. J. Martinez, B. Hockey, C. B. Montgomery, and S. Semancik, Langmuir 21, 7937 (2005).

${ }^{10}$ Y. C. Wang and R. S. Lakes, J. Compos. Mater. 39, 1645 (2005).

${ }^{11}$ S. R. White, N. R. Sottos, P. H. Geubelle, J. S. Moore, M. R. Kessler, S. R. Sriram, E. N. Brown, and S. Viswanatan, Nature 409, 794 (2001).

${ }^{12}$ G. N. Greaves, A. L. Greer, R. S. Lakes, and T. Rouxel, Nature. Mater. 10, 823 (2011).

${ }^{13}$ N. Chan and K. E. Evans, J. Cell. Plast. 34, 231 (1998).

${ }^{14}$ J. Zhang, D. Jiang, F. Scarpa, and H. Peng, Composites Part A 55, 188 (2013).

${ }^{15}$ J. P. M. Whitty, A. Alderson, P. Myler, and B. Kandola, Composites Part A 34, 525 (2003).

${ }^{16}$ L. Chen, C. Liu, J. Wang, W. Zhang, C. Hu, and S. Fan, Appl. Phys. Lett. 94, 253111 (2009).

${ }^{17}$ K. E. Evans and A. Alderson, Adv. Mater. 12, 617 (2000).

${ }^{18}$ R. Lakes, Adv. Mater. 5, 293 (1993).

${ }^{19}$ Y. Hou, Y. H. Tai, C. Lira, F. Scarpa, J. R. Yates, and B. Gu, Composites Part A 49, 119 (2013).

${ }^{20}$ Z. Ma, H. Bian, C. Sun, G. M. Hulbert, K. Bishnoi, and F. Rostam-Abadi, Proceedings of the 2010 NDIA Ground Vehicle Systems Engineering and Technology Symposium, Michigan, USA, 17-19 August 2010.

${ }^{21}$ R. Lakes, Science 235, 1038 (1987).

${ }^{22}$ F. Scarpa, J. R. Yates, L. G. Ciffo, and S. Patsias, J. Mech. Eng. Sci. 216, 1153 (2002).

${ }^{23}$ J. R. Wright, M. R. Sloan, and K. E. Evans, J. Appl. Phys. 108, 044905 (2010).

${ }^{24}$ K. Krieger, Nature 488, 146 (2012).

${ }^{25}$ X. Hou, H. Hu, and V. Silberschmidt, Compos. Sci. Technol. 72, 1848 (2012). 